\title{
Process optimization for synthesis of Anti-Tuberculosis drug catalyzed by fluor apatite supported potassium fluoride
}

\author{
Younes Abrouki ${ }^{1 \text {, }}$, Abdelkader Anouzla ${ }^{1}$, Hayat Loukili ${ }^{1}$, Rabiaâ Lotfi ${ }^{1}$, Ahmed Rayadh ${ }^{1}$, \\ Abdellah Bahlaoui ${ }^{1}$, Saïd Sebti ${ }^{2}$, Driss Zakarya ${ }^{1}$, Mohamed Zahouily ${ }^{1}$ \\ ${ }^{1}$ Department of Research, Faculty of Science and Technology, University Hassan II, Mohammedia, Morocco \\ ${ }^{2}$ Department of Chemistry, Faculty of Science Ben M'Sik, University Hassan II, Casablanca, Morocco
}

Email address:

abroukiyounes@yahoo.fr(Y. Abrouki)

To cite this article:

Younes Abrouki, Abdelkader Anouzla, Hayat Loukili, Rabiaâ Lotfi, Ahmed Rayadh, Abdellah Bahlaoui, Saïd Sebti, Driss Zakarya, Mohamed Zahouily. Process Optimization for Synthesis of Anti-Tuberculosis Drug Catalyzed by Fluor apatite Supported Potassium Fluoride. American Journal of Theoretical and Applied Statistics. Vol. 2, No. 3, 2013, pp. 81-86.

doi: $10.11648 /$ j.ajtas.20130203.16

\begin{abstract}
The optimization of the synthesis of anti-tuberculosis drug by thia-Michael addition between thiophenol and chalcone catalyzed using activated Fluorapatite supported potassium fluoride (KF/FAP) was studied using a 2 block central composite design including 4 factors (reaction time, solvent volume, catalyst weight and impregnation ratio). The high reactivity and regioelectivity of our catalyst coupled with their ease of use and reduced environmental problems makes them attractive alternatives to homogeneous basic reagents.
\end{abstract}

Keywords: Anti-Tuberculosis Drug, Fluorapatite Supported Potassium Fluoride, Heterogeneous Catalyst, Response Optimization; Central Composite Design

\section{Introduction}

In the last few years, tuberculosis (TB), caused by Mycobacterium tuberculosis, has again become one of the most dangerous and lethal infectious diseases [1]. According to the new WHO report in 2010, there were an estimated 8.8 million new cases of TB, 1.45 million TB deaths and more than two billion people infected with M. tuberculosis, of which approximately $10 \%$ will most likely become ill during their life [2]. This situation is due to HIV infection (1.1 million of all TB causes and 0.35 million of TB deaths are in people who are HIV positive), poor compliance of patients, population migration and increased drug resistance [3].

Recently, the Czech Authors [4] are showed that the 1, 3-Diphenyl-3-phenylsulfanyl-propan-1-one compound presented an anti-Mycobacterium tuberculosis. The conjugate addition of thiophenol to chalcone is a convenient route for synthesis of this sulfanyl organic compound [5].

In classic methods, this reaction catalyzed by strong bases such as alkali metal alkoxides [6], hydroxides [7] and amines [8]. The employment of these strong bases and acids in these reactions [9], however, leads to two main problems affecting the environment; the necessity to dispose of huge amounts of organic waste due to formation of undesirable side products resulting from polymerization, bis-addition and self condensation, and total dissolved salts formed following the neutralization of soluble bases with acids. The replacement of liquid basic catalysts by solid bases in the synthesis of fine and intermediate organic chemicals allows one to avoid corrosion and environmental problems [10].

In view of this, the aim of the present study is investigate the application of experiments design for modeling and optimization of the synthesis of anti- tuberculosis drug 1,3-Diphenyl-3-phenylsulfanyl-propan-1-one catalyzed using activated Fluorapatite supported by potassium fluoride.

\section{Materials and Methods}

\subsection{Chemicals and Instrumentations}

All commercial reagents and solvents were used without further purification. X-ray diffraction (XRD) patterns of the 
catalysts were obtained on a Philips 1710 diffractometer using $\mathrm{Cu}-\mathrm{K} \alpha$ radiation. Surface areas were determined at $77 \mathrm{~K}$ using a Coulter SA 31000 instrument with an automated gas volumetric method employing nitrogen as the adsorbate. NMR spectra were recorded on a Bruker ARX 300 spectrometer. Mass spectra were recorded on a VG Autospec spectrometer. FTIR spectra were recorded on an ATI Mattson-Genesis Series spectrophotometer using the $\mathrm{KBr}$ disc method.

\subsection{Preparation and Characterization of Catalysts}

The Fluorapatite catalyst (FAP) was prepared by the co-precipitation method [11], using the following reaction:

$$
\begin{gathered}
6\left(\mathrm{NH}_{4}\right)_{2} \mathrm{HPO}_{4}+10 \mathrm{Ca}\left(\mathrm{NO}_{3}\right)_{2}+2 \mathrm{NH}_{4} \mathrm{~F}+6 \mathrm{NH}_{4} \mathrm{OH} \\
\rightarrow \mathrm{Ca}_{10}\left(\mathrm{PO}_{4}\right)_{6} \mathrm{~F}_{2}+20 \mathrm{NH}_{4}\left(\mathrm{NO}_{3}\right)+6 \mathrm{H}_{2} \mathrm{O}
\end{gathered}
$$

The activated Fluorapatite supported by potassium fluoride catalyst (KF/FAP) has been prepared by impregnation method [12]. The ratio of impregnation $(R I)$ for KF/FAP catalyst was calculated according to equation (1):

$$
\mathrm{RI}=(\text { Weight } \text { of } \mathrm{KF}) /(\text { Weight of FAP })
$$

The analysis of the catalysts FAP and KF/FAP with X-ray diffraction gives the diffraction patterns almost identical. The comparison of images by scanning electron micrograph for FAP with KF/FAP shows a modification in the morphology of the doped material.

These results indicate that the structure of the apatite remains unchanged and that the potassium fluoride interacted with the surface of the support of catalyst.

\subsection{General Procedure}

The general procedure is reported in Figure 1, as follows: To a flask containing an equimolar mixture $(1 \mathrm{mmol})$ of chalcone 1 and thiophenol 2 in methanol, the KF/FAP or FAP was added and the mixture was stirred at room temperature. The catalyst was filtered, washed with dichloromethane and the filtrate was concentrated under reduced pressure.

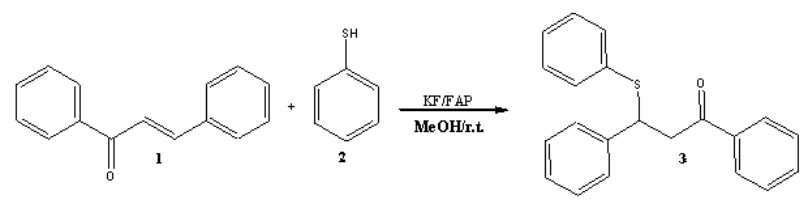

Figure 1. Synthesis of anti-tuberculosis drug

The crude product 3 was purified by recrystallization. The product was analyzed by ${ }^{1} \mathrm{H},{ }^{13} \mathrm{C}$ NMR and IR spectrometry.

\subsection{Statistical Analysis}

The synthesis of anti-tuberculosis drug 1,3 -Diphenyl-3-phenylsulfanyl-propan-1-one from the
thia-Michael addition between chalcone and thiophenol catalyzed using KF/FAP catalyst was carried out and optimized using the Central Composite Design (CCD) and Response Surface Methodology (RSM) [13]. CCD helps in investigating linear, quadratic, cubic and cross-product effects of the four reaction condition variables on the reaction yield. The four independent variables studied were reaction time $\left(\mathrm{X}_{1}\right)$, solvent volume $\left(\mathrm{X}_{2}\right)$, catalyst weight $\left(\mathrm{X}_{3}\right)$ and impregnation ratio $\left(\mathrm{X}_{4}\right)$. Table 1 lists the range and levels of the four independent variables studied.

Table 1. Study field and coded factors

\begin{tabular}{ccccc}
\hline $\begin{array}{c}\text { Un-coded variables } \\
\mathbf{x}_{\mathbf{1}}, \mathbf{x}_{\mathbf{2}}, \mathbf{x}_{\mathbf{3}} \text { and } \mathbf{x}_{\mathbf{4}}\end{array}$ & Unit & \multicolumn{4}{c}{ Coded variables $\mathbf{X}_{\mathbf{1}}, \mathbf{X}_{\mathbf{2}}, \mathbf{X}_{\mathbf{3}}$ and $\mathbf{X}_{\mathbf{4}}$} \\
\hline $\mathrm{x}_{1}=$ Reaction time & $\min$ & 3 & 4 & $\mathbf{+ 1}$ \\
$\mathrm{x}_{2}=$ Solvent volume & $\mathrm{mL}$ & 2 & 4 & 5 \\
$\mathrm{x}_{3}=$ Catalyst weight & $\mathrm{mg}$ & 80 & 120 & 6 \\
$\mathrm{x}_{4}=$ Impregnation ratio & & $1 / 24$ & $1 / 12$ & $1 / 8$ \\
\hline
\end{tabular}

The experimental data obtained by following the above procedure were analyzed by the response surface methodology using the following second-order polynomial equation:

$$
\begin{aligned}
& Y=\beta_{0}+\beta_{1} X_{1}+\beta_{2} X_{2}+\beta_{3} X_{3}+\beta_{4} X_{4} \\
& +\beta_{11} X_{11}+\beta_{22} X_{22}+\beta_{33} X_{33}+\beta_{44} X_{44} \\
& +\beta_{12} X_{1} X_{2}+\beta_{13} X_{1} X_{3}+\beta_{14} X_{1} X_{4} \\
& +\beta_{23} X_{2} X_{3}+\beta_{24} X_{2} X_{4}+\beta_{34} X_{3} X_{4}
\end{aligned}
$$

Where $\mathrm{Y}$ is the response (reaction yield); $\mathrm{X}_{i}$ and $\mathrm{X}_{j}$ are the coded independent variables and $\beta_{0}, \beta_{i}, \beta_{i i}$ and $\beta_{i j}$ are intercept, linear, quadratic and interaction constant coefficients, respectively. The software stat graphics-Plus was used for regression analysis and analysis of variance (ANOVA). Response surfaces and contour plots were developed using the fitted quadratic polynomial equation obtained from regression analysis, holding two of the independent variables at a constant value corresponding to the stationary point and changing the other two variables. Confirmatory experiment was carried out to validate the equation, using combinations of independent variables which were not part of the original experimental design but within the experimental region.

\section{Results and Discussion}

\subsection{Response Surface Methodology}

By using a central composite design (CCD), a correlation between the reaction condition variables to the reaction yield was developed. Table 2 shows the complete design matrix and reaction yield at various reaction condition variables.

Selection of the levels was based on our preliminary 
study. The value of $\alpha$ for this CCD was fixed at two. The complete design matrix of the experiments conducted and results are given in Table 2.

All variables at zero level constitute to the center points and the combination of each of the variables at either it's lowest $(-2.0)$ level or highest $(+2.0)$ level with the order variables at zero level constitute the axial points. The experiment sequence was randomized in order to minimize the effects of the uncontrolled factors.

In table 2 we can show, the reaction yield obtained was in the range from 32 to $95 \%$. Experimental error was determined from run 25 to 26 at the centre point of the design.

Table 2. Experimental design and results

\begin{tabular}{|c|c|c|c|c|c|c|c|c|c|c|}
\hline \multirow{2}{*}{$\begin{array}{c}\text { Standard } \\
\text { order }\end{array}$} & \multirow{2}{*}{$\begin{array}{l}\text { Run } \\
\text { order }\end{array}$} & \multicolumn{4}{|c|}{ Coded variables } & \multicolumn{4}{|c|}{ Un-coded variables } & \multirow{2}{*}{ Reaction yield } \\
\hline & & $\mathbf{X}_{1}$ & $\mathbf{X}_{2}$ & $\mathbf{X}_{3}$ & $\mathbf{X}_{4}$ & $\mathbf{x}_{1}$ & $\mathbf{x}_{2}$ & $\mathbf{x}_{3}$ & $\mathbf{x}_{4}$ & \\
\hline 1 & 4 & +1 & +1 & +1 & +1 & 5 & 6 & 160 & $1 / 8$ & 83 \\
\hline 2 & 19 & +1 & +1 & +1 & -1 & 5 & 6 & 160 & $1 / 24$ & 54 \\
\hline 3 & 1 & +1 & +1 & -1 & +1 & 5 & 6 & 80 & $1 / 8$ & 76 \\
\hline 4 & 20 & +1 & +1 & -1 & -1 & 5 & 6 & 80 & $1 / 24$ & 47 \\
\hline 5 & 12 & +1 & -1 & +1 & +1 & 5 & 2 & 160 & $1 / 8$ & 94 \\
\hline 6 & 2 & +1 & -1 & +1 & -1 & 5 & 2 & 160 & $1 / 24$ & 64 \\
\hline 7 & 24 & +1 & -1 & -1 & +1 & 5 & 2 & 80 & $1 / 8$ & 95 \\
\hline 8 & 26 & +1 & -1 & -1 & -1 & 5 & 2 & 80 & $1 / 24$ & 64 \\
\hline 9 & 3 & -1 & +1 & +1 & +1 & 3 & 6 & 160 & $1 / 8$ & 62 \\
\hline 10 & 25 & -1 & +1 & +1 & -1 & 3 & 6 & 160 & $1 / 24$ & 38 \\
\hline 11 & 18 & -1 & +1 & -1 & +1 & 3 & 6 & 80 & $1 / 8$ & 58 \\
\hline 12 & 6 & -1 & +1 & -1 & -1 & 3 & 6 & 80 & $1 / 24$ & 33 \\
\hline 13 & 15 & -1 & -1 & +1 & +1 & 3 & 2 & 160 & $1 / 8$ & 71 \\
\hline 14 & 9 & -1 & -1 & +1 & -1 & 3 & 2 & 160 & $1 / 24$ & 46 \\
\hline 15 & 16 & -1 & -1 & -1 & +1 & 3 & 2 & 80 & $1 / 8$ & 73 \\
\hline 16 & 5 & -1 & -1 & -1 & -1 & 3 & 2 & 80 & $1 / 24$ & 48 \\
\hline 17 & 10 & +2 & 0 & 0 & 0 & 6 & 4 & 120 & $1 / 12$ & 81 \\
\hline 18 & 8 & -2 & 0 & 0 & 0 & 2 & 4 & 120 & $1 / 12$ & 53 \\
\hline
\end{tabular}




\begin{tabular}{|c|c|c|c|c|c|c|c|c|c|c|}
\hline 19 & 21 & 0 & +2 & 0 & 0 & 4 & 8 & 120 & $1 / 12$ & 44 \\
\hline 20 & 11 & 0 & -2 & 0 & 0 & 4 & 0 & 120 & $1 / 12$ & 61 \\
\hline 21 & 23 & 0 & 0 & +2 & 0 & 4 & 4 & 200 & $1 / 12$ & 64 \\
\hline 22 & 7 & 0 & 0 & -2 & 0 & 4 & 4 & 40 & $1 / 12$ & 36 \\
\hline 23 & 17 & 0 & 0 & 0 & +2 & 4 & 4 & 120 & $1 / 6$ & 95 \\
\hline 24 & 14 & 0 & 0 & 0 & -2 & 4 & 4 & 120 & 0 & 32 \\
\hline 25 & 22 & 0 & 0 & 0 & 0 & 4 & 4 & 120 & $1 / 12$ & 73 \\
\hline 26 & 13 & 0 & 0 & 0 & 0 & 4 & 4 & 120 & $1 / 12$ & 71 \\
\hline
\end{tabular}

\subsection{Regression Analysis}

A regression analysis was performed to fit the response function and predict the outcome of reaction yield with a simple equation. The model is expressed by Eq. (3) which takes their coded value.

$$
\begin{gathered}
Y=72+8.5 X_{1}-5.75 X_{2}+3.08 X_{3}+14.33 X_{4} \\
-0.48 X_{1}^{2}-4.12 X_{2}^{2}-4.73 X_{3}^{2}-1.36 X_{4}^{2} \\
-0.63 X_{1} X_{2}+0.5 X_{1} X_{3}+1.25 X_{1} X_{4} \\
+1.75 X_{2} X_{3}-0.25 X_{2} X_{4}-0.13 X_{3} X_{4}
\end{gathered}
$$

The summary of the analysis of variance (ANOVA) result is shown in Table 3.

The regressors or term incorporated in the model are those statistically tested to be significant. The 'Prob $>F$ ' value indicates the probability equals the proportion of the area under the curve of the $F$-distribution that lies beyond the observed $F$ value. The small probability values called for the rejection of the null hypothesis, in other words, the particular term significantly affected the measured response of the system. In this case, the linear terms $\left(X_{1}, X_{2}, X_{3}\right.$ and $\left.X_{4}\right)$ and the squared terms $\left(X_{2}^{2}\right.$ and $\left.X_{3}^{2}\right)$ were significant model terms whereas the squared terms $\left(X_{1}^{2}\right.$ and $\left.X_{4}^{2}\right)$ and the interaction terms $\left(X_{1} X_{2}, X_{1} X_{3}, X_{1} X_{4}, X_{2} X_{3}, X_{2} X_{4}\right.$ and $\left.X_{3} X_{4}\right)$ were insignificant to the response. On the other hand, from this ANOVA the model F-value of 599.46 implied that the model was significant. Values of Prob. > F less than 0.01 indicated that the model terms was significant.

The final empirical model in term of coded factors after excluding the insignificant terms for reaction yield is shown in Eq. (4):

$$
Y=72+8.5 X_{1}-5.75 X_{2}+3.08 X_{3}+14.33 X_{4}-4.12 X_{2}^{2}-4.73 X_{3}^{2}
$$

Table 3. Regression variance analysis for the model

\begin{tabular}{ccccccc}
\hline Source of variation & Coefficient & Sum of Squares & $\mathbf{v}$ & Mean square & $\mathbf{F}_{\text {exp }}$ & Significance test \\
\hline Regression & - & 8392.38 & 14 & 0599.4600 & 017.40 & $* * *$ \\
$\beta_{0}$ & 72.00 & - & 1 & - & - & \\
$\beta_{1}$ & 08.50 & 1734.0000 & 1 & 1734.0000 & 050.33 & $* * *$ \\
$\beta_{2}$ & -05.75 & 0793.5000 & 1 & 0793.5000 & 023.03 & $* * *$ \\
$\beta_{3}$ & 03.08 & 0228.1670 & 1 & 0228.1670 & 006.62 & $*$ \\
$\beta_{4}$ & 14.33 & 4930.6700 & 1 & 4930.6700 & 143.11 & $* * *$ \\
$\beta_{11}$ & -00.48 & 0004.0076 & 1 & 0004.0076 & 000.12 & NS \\
$\beta_{12}$ & -00.63 & 0006.2500 & 1 & 0006.2500 & 000.18 & NS \\
$\beta_{13}$ & 00.50 & 0004.0000 & 1 & 0004.0000 & 000.12 & NS \\
$\beta_{14}$ & 01.25 & 0025.0000 & 1 & 0025.0000 & 000.73 & NS \\
$\beta_{22}$ & -04.12 & 0294.0080 & 1 & 0294.0080 & 008.53 & $* *$
\end{tabular}




\begin{tabular}{ccccccc}
$\beta_{23}$ & 01.75 & 0049.0000 & 1 & 0049.0000 & 001.42 & NS \\
$\beta_{24}$ & -00.25 & 0001.0000 & 1 & 0001.0000 & 000.03 & NS \\
$\beta_{33}$ & -04.73 & 0390.3710 & 1 & 0390.3710 & 011.33 & $* * *$ \\
$\beta_{34}$ & -00.13 & 0000.2500 & 1 & 0000.2500 & 000.01 & NS \\
$\beta_{44}$ & -01.36 & 0032.0076 & 1 & 0032.0076 & 000.93 & NS \\
Residue & - & 0379.0000 & 11 & 0034.4600 & & \\
Total & - & 8771.3800 & 25 & - & & \\
\hline
\end{tabular}

$*_{* *}: \mathrm{p} \leq 0,01 ; * *: \mathrm{p} \leq 0,025 ; *: \mathrm{p} \leq 0,05 ;$ NS $:$ No significant.

The coefficient of determination, $R^{2}$ for the model was $95.68 \%$. This indicates that only $4.32 \%$ of the total variability was not explained by the regressors in the model. The high $R^{2}$ value specifies that the model obtained will be able to give a convincingly good estimate of response of the system in the range studied.

\subsection{Model Analysis}

The influence of each independent variable on the response is shown in Figure 2.

This figure shows that the reaction time and the impregnation ratio have a positive effect on the reaction yield, while the solvent volume has a negative effect on the response. The catalyst weight also has a positive effect on the reaction yield, but by looking at this figure, we see that it has a negative effect in the positive area, which can be explained by the negative effect of the interaction of the squared term.

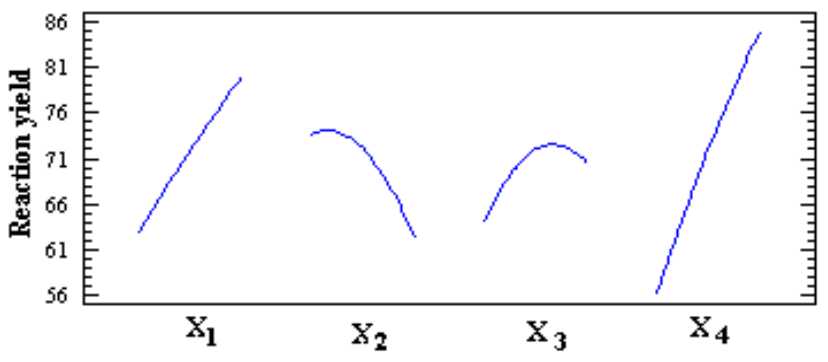

Figure 2. Main effects plot for reaction yield.

The geometric representation of the reaction yield according to the catalyst weight and the solvent volume is shown in Figure 3.

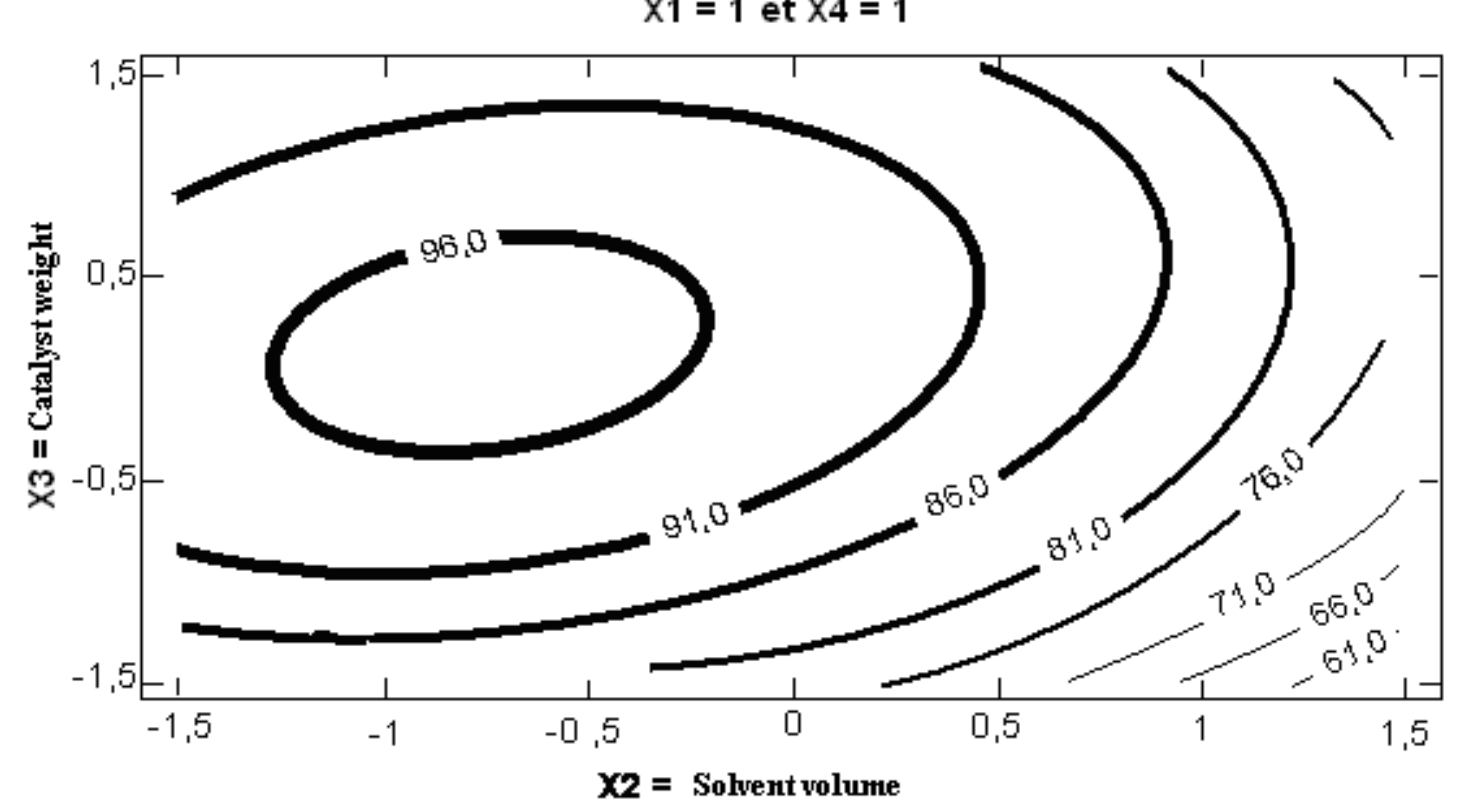

Figure 3. Contour plot of the combined effects of catalyst weight and solvent volume on the reaction yield at fixed reaction time and impregnation ratio

Analysis of the contour plot shows that for a fixed solvent volume, the increase in catalyst weight increases the reaction yield. Moreover, for a fixed catalyst weight, increasing the solvent volume decreases the reaction yield 
in the experimental field.

\subsection{Process Optimization}

Basically, the CCD was able to optimally design the desired response of the system based on the model obtained and the input criteria. The optimization of reaction yield was carried out based on the four variables which were in the range of experimental runs. The software predicted that optimized conditions for reaction yield were obtained when the reaction time, solvent volume, catalyst weight and impregnation ratio, were at $5 \mathrm{~min}, 2 \mathrm{~mL}, 110 \mathrm{mg}, 1 / 2$, respectively with predicted reaction yield of $96 \%$. The experimental reaction yield was $94 \%$. This shows that the experimental value obtained was in good agreement with the value calculated from the model.

\section{Conclusion}

In summary, the optimization for synthesis of 1 , 3-Diphenyl-3-phenylsulfanyl-propan-1-one catalyzed by activated Fluorapatite supported by potassium fluoride has been studied using central composite design. The model equation for the optimization of the reaction conditions for this synthesis was established. From this equation it was possible to forecast the optimal reaction conditions for synthesis of anti-tuberculosis drug in high yield.

This process bring advantages such as high catalytic activity and selectivity under mild reaction conditions, easy separation of the catalyst by simple filtration, use of non-toxic and inexpensive catalysts and especially, elimination of salts and by-product pollutants. This new solid base catalyst becomes then a practical alternative to soluble bases.

\section{Acknowledgements}

We are grateful to Mr. Hamid Dhimane and Mr. Marc David of Laboratoire de Chimie et Biochimie Pharmacologiques et Toxicologiques, Université René Descartes Paris-France, for his help with data analysis.

\section{References}

[1] J. Kozic, E. Novotná, M. Volková, J. Stolaríková, F. Trejtnar, and J. Vinsová, Synthesis and in vitro antimycobacterial activity of 2-methoxybenzanilides and their thioxo analogues, Euro. J. Med. Chem., 56, (2012), pp. 387-395.

[2] WHO report, Global Tuberculosis Control (2011).http://www.who.int/tb/publications/global report/2011/gtbr11 full.pdf.
[3] S.T. Cole, G. Riccardi, New tuberculosis drugs on the horizon, Curr. Opin. Microbiol. 14 (2011), pp. 570-576.

[4] M. Chlupáčová, P. Kubanová, V. Opletalová, V. Buchta, The Importance of the Enone-moiety for Antimycobacterial and Antifungal Properties of Chalcones, Published in: Proceedings of the 3rd International Symposium on Natural Drugs, Naples, 2-4 October 2003. Borelli, F., Capasso, F., Milic, N., Russo, A. (Eds.). Universita degli Studi di Napoli Federico II, Naples - Indena, Milano 2003, pp. 133-135.

[5] Y. Abrouki, M. Zahouily, A. Rayadh, B. Bahlaouan, S. Sebti, A natural phosphate and doped-catalyzed Michael addition of mercaptans to $\alpha, \beta$-unsaturated carbonyl compounds, Tet. Lett., 43, (2002), pp. 8951-8953.

[6] M. Ceylan, M. B. Gürdere, H. Gezegen, Y. Budak, Potassium-tertiary butoxide-assisted Addition of thioglycolic acid to chalcone derivatives under solvent-free conditions, Synt. Commun., 40 (2010), pp. 2598-2606.

[7] J. T. Li, Y. Cui, G. F. Chen, Z. L. Cheng, T. S. Li, Michael Addition Catalyzed by Potassium Hydroxide Under Ultrasound, Synthetic Comm., 33, (2003), pp. 353-359.

[8] H. Hiemstra, H. Wynberg, Addition of aromatic thiols to conjugated cycloalkenones, catalyzed by chiral $\beta$-hydroxy amines: A mechanistic study of homogeneous catalytic asymmetric synthesis, J. Am. Chem. Soc., 103 (1981), pp. 417-430.

[9] T. C. Wabnitz, J. B. Spencer, A general Brønsted Acid-Catalyzed Hetero-Michael Addition of Nitrogen, Oxygen, and Sulfur Nucleophiles, Org. Lett., 12, (2003), pp. 2141-2144.

[10] Y. Riadi, R. Mamouni, Y. Abrouki, M. El Haddad, N. Saffaj, S. El Antri, S. Routier, G. Guillaumet, S. Lazar, Animal Bone Meal (ABM): A Novel Natural Catalyst for Thia-Michael Addition, Tet. Lett., 51, (2010), pp. 6715-6717.

[11] M. Zahouily, Y. Abrouki, A. Rayadh, S. Sebti, H. Dhimane, and M. David, Fluorapatite: efficient catalyst for the Michael addition, Tet. Lett. 44, (2003), pp. 2463-2465.

[12] Smahi, A. Solhy, H. El Badaoui, A. Amoukal, A. Tikad, M. Maizi, and S. Sebti, Potassium fluoride doped fluorapatite and hydroxyapatite as new catalysts in organic synthesis Appl. Catal. A. Gen., 250, (2003), pp. 151-159.

[13] A. Anouzla, Y. Abrouki, S. Souabi, M. Safi, H. Rhbal, Colour and COD removal of disperse Dye solution by a novel Coagulant: Application of statistical Design for the optimization and Regression analysis, J. Hazard. Mater., 166 (2009), pp. 1302-1306. 